\title{
Value of endoscopic ultrasound guided fine needle aspiration biopsy in the diagnosis of solid pancreatic masses
}

\author{
M Voss, P Hammel, G Molas, L Palazzo, A Dancour, D O’Toole, B Terris, C Degott,
} P Bernades, P Ruszniewski

\begin{abstract}
Aim-To assess the feasibility and diagnostic accuracy of endoscopic ultrasound guided fine needle biopsy (EUS-FNAB) in patients with solid pancreatic masses. Methods-Ninety nine consecutive patients with pancreatic masses were studied. Histological findings obtained by EUS-FNAB were compared with the final diagnosis assessed by surgery, biopsy of other tumour site or at postmortem examination, or by using a combination of clinical course, imaging features, and tumour markers.
\end{abstract}

Results-EUS-FNAB was feasible in 90 patients (adenocarcinomas, $n=59$; neuroendocrine tumours, $n=15$; various neoplasms, $n=6$; pancreatitis, $n=10$ ), and analysable material was obtained in 73 . Tumour size ( $\geqslant$ or $<25 \mathrm{~mm}$ in diameter) did not influence the ability to obtain informative biopsy samples. Diagnostic accuracy was $74.4 \%$ (adenocarcinomas, $81.4 \%$; neuroendocrine tumours, $46.7 \%$; other lesions, $75 \%$; $<<0.02$ ). Overall, the diagnostic yield in all 99 patients was $68 \%$. Successful biopsies were performed in six patients with portal hypertension. Minor complications (moderate bleeding or pain) occurred in $5 \%$ of cases.

Conclusions-EUS-FNAB is a useful and safe method for the investigation of pancreatic masses, with a high feasibility rate even when lesions are small. Overall diagnostic accuracy of EUS-FNAB seems to

Medical-Surgica

Federation of

Hepato-Gastroenterology, depend on the tumour type.

Beaujon Hospital, （Gut 2000;46:244-249)

Clichy, France

$M$ Voss

P Hammel

L Palazzo

A Dancour

D O'Toole

P Bernades

P Ruszniewski

Department of

Pathology, Beaujon

Hospital

G Molas

B Terris

C Degott

Correspondence to:

Dr P Hammel, Service de

Gastroentérologie, hôpital

Beaujon, 92110 Clichy,

France.

Accepted for publication 9 August 1999 cal diagnosis is important. In a retrospective series published in 1982 by Lee, ${ }^{4}$ examination of the resected specimen disclosed benign disease in up to $25 \%$ of surgical resections performed without a diagnosis before the operation. Investigations before surgery should provide accurate staging and tissue diagnosis with a low morbidity and without compromising resectability.

Since its introduction in the $1980 \mathrm{~s},{ }^{56}$ endoscopic ultrasound (EUS) has been established as an accurate imaging procedure in the diagnosis and staging of pancreatic tumours. ${ }^{7-9}$ In patients with operable conditions, EUS is a third line investigation after ultrasound and spiral computed tomography (CT) scan, where these investigations have not detected obvious criteria for non-resectability. ${ }^{9}$ A combination of EUS and endoscopic ultrasound guided fine needle aspiration biopsy (EUS-FNAB) in patients with pancreatic tumours allows staging and the possibility of a histological diagnosis during the same procedure. The technique and applications of EUS-FNAB have been previously described. ${ }^{10-12}$ In contrast with cytology alone, the possibility of obtaining a biopsy tissue core sample using this procedure is desirable, allowing analysis of tissue structure with serial sections and the possibility of immunostaining, thus improving the diagnostic precision.

Our study aimed to assess the feasibility and diagnostic accuracy of EUS-FNAB in a series of 99 consecutive patients with pancreatic masses.

\section{Patients and methods}

INCLUSION CRITERIA

The records of 99 consecutive patients undergoing EUS-FNAB for the diagnosis of solid pancreatic masses at Beaujon Hospital between January 1995 and March 1998 were analysed. EUS-FNAB was not performed in patients with easily accessible metastases because histological diagnosis could be achieved using simpler methods - for example, transparietal biopsy of hepatic metastases. As the study was designed to assess both the feasibility and accuracy of EUS-FNAB in patients with pancreatic masses, several patients with large tumours were included, although transparietal pancreatic biopsy would have been technically possible in some of them. In five patients who

Abbreviations used in this paper: EUS-FNAB, endoscopic ultrasound guided fine needle aspiration biopsy; CT, computed tomography. 
underwent a second EUS-FNAB procedure after an initial non-diagnostic sample, only the results of the second procedure were analysed.

When histological analysis provided an unequivocal result, it was considered to be the definitive diagnosis. When histological diagnosis was uncertain, a combination of other criteria such as clinical course, imaging features, serum carcinoembryonic antigen, and carbohydrate tumour antigen 19.9 was used to assess the diagnosis of exocrine tumours. When a diagnosis of neuroendocrine tumour was suspected, serum peptide measurements and indium In-111 pentetreotide scintigraphy were performed. A benign disease was suspected when there were no changes in imaging studies over a minimum of six months and when the clinical course was favourable.

\section{EXCLUSION CRITERIA}

Patients with purely cystic tumours of the pancreas or pseudocysts were excluded because the diagnostic approach in these patients is different, involving cytocentrifugation or analysis of cystic fluid for biochemical and tumour markers rather than core needle biopsy even if informative tumour epithelium specimen can occasionally be obtained. ${ }^{13-15}$

\section{GENERAL STUDY DESIGN}

Data were recorded with special attention to clinical presentation and EUS findings, in particular the size and site of the tumour, the presence of calcifications, and portal hypertension. The number of needle passes, complications after the procedure, and the final diagnosis were noted.

\section{ENDOSCOPIC METHODS}

All patients had general anaesthesia. An initial diagnostic EUS was performed with an Olympus EUM $20360^{\circ}$ apparatus (Olympus, Rungis, France) for staging and localisation of the tumour. The instrument was then changed for a Pentax FG 32 UA $120^{\circ}$ (Pentax, les Ulis, France) sectorial scanner with an operating port. The needle-catheter system was inserted through the working channel of the endoscope and the needle advanced into the target lesion using a 22 gauge needle under ultrasound control (figs 1 and 2). After removal of the stylet, a $30 \mathrm{ml}$ syringe with automatic suction priming was applied to the biopsy channel and the needle was carefully advanced back and forth within the target lesion. This procedure was repeated, with suction applied for up to two to three minutes. Aspiration biopsy was terminated if blood became visible in the syringe. After suction release, the catheter system was removed and the biopsy sample placed in Bouin's solution. The procedure was repeated until a core of tissue had been obtained or a maximum of three needle passages. The biopsy site was examined for evidence of bleeding, and patients were observed for at least four hours before discharge. Passage was transduodenal for lesions in the head/uncinate process of the pancreas and transgastric through the lesser sac for lesions in the body and tail.

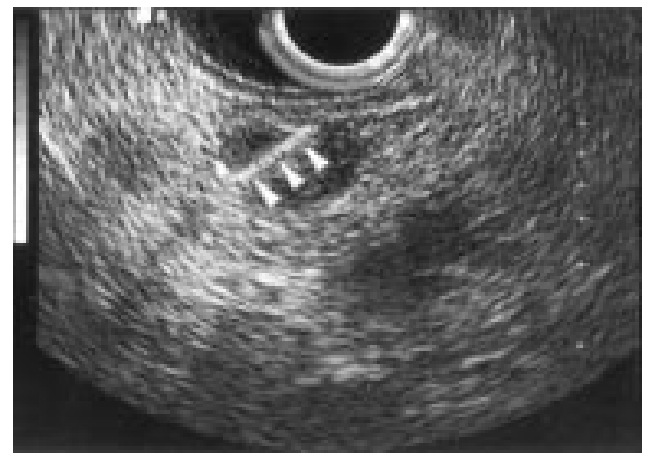

Figure 1 Example of endoscopic ultrasound guided fine needle aspiration biopsy (EUS-FNAB) in a

neuroendocrine tumour of the body of the pancreas measuring $15 \mathrm{~mm}$ in diameter. The arrowheads show the position of the needle.

HISTOLOGICAL AND CYTOLOGICAL METHODS Fragments were fixed in Bouin's solution, paraffin embedded and sectioned at a minimum of six levels, and stained with haematoxylin-eosin and safran. Specific stains were used: periodic acid/Schiff with amylase glycogen digestion and alcian blue for exocrine tumour; chromogranin, synaptophysin, and neurone-specific enolase techniques for neuroendocrine tumour. The epithelial KL1 and EMA markers and the macrophage KP1 marker were used to differentiate between epithelial cells and macrophages. In addition to microbiopsy, when abundant material was available, some of the aspirated material was sprayed on to glass slides for cytological analysis. In the case of fluid aspiration, cell suspensions obtained by
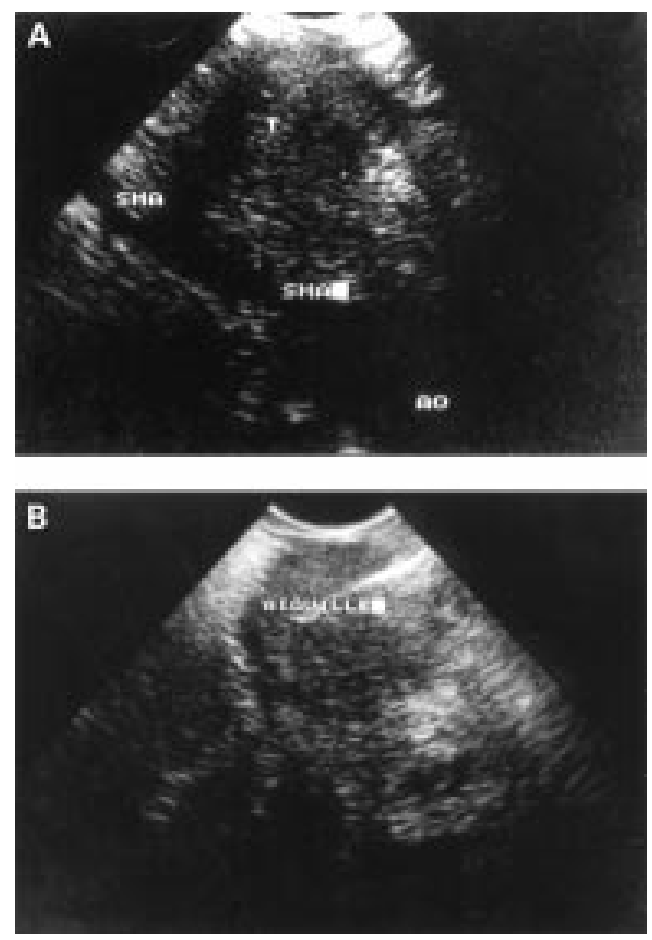

Figure 2 (A) Endoscopic ultrasound findings strongly suggestive of a malignant tumour of the pancreatic head (T). Because of invasion of the superior mesenteric artery (SMA), this tumour was not suitable for surgical resection. (B) Endoscopic ultrasound guided fine needle aspiration biopsy of this tumour gave the diagnosis of adenocarcinoma (aiguille $=$ needle). 
Table 1 Comparison between endoscopic ultrasound fine needle aspiration needle biopsy (EUS-FNAB) histological findings and final diagnosis in 90 patients with pancreatic masses who underwent successful biopsy

\begin{tabular}{lllll}
\hline & & \multicolumn{2}{l}{$\begin{array}{l}\text { Result after histological examination of } \\
\text { EUS-FNAB samples }\end{array}$} \\
\cline { 3 - 5 } & $\begin{array}{c}\text { Final } \\
\text { diagnosis }\end{array}$ & $\begin{array}{l}\text { Certain/strongly } \\
\text { suspected }\end{array}$ & $\begin{array}{l}\text { False } \\
\text { negative }\end{array}$ & False positive \\
\hline Pancreatic neoplasms & & & & \\
$\quad$ Adenocarcinoma & 59 & $43 / 5$ & $11(8) \dagger$ & 1 \\
$\quad$ Neuroendocrine tumour & 15 & $6 / 1$ & $8(7)$ & 0 \\
$\quad$ Various & 5 & $2 / 2$ & 1 & 0 \\
$\quad$ IPMT (benign) & 1 & $0 / 1$ & 0 & 0 \\
$\quad$ Cystic and papillary tumour & 10 & $4 / 3$ & $2(2)$ & 0 \\
Pancreatitis & 90 & 67 & 22 & 1 \\
Total & & & & \\
\hline
\end{tabular}

^Including one cystadenocarcinoma, five malignant papillary mucinous tumours (IPMT), one oestoclast-like carcinoma, and two pancreatic metastases (from primary breast and renal cell cancer).

†Including non-informative samples.

centrifugation were analysed. May GrunwaldGiemsa stain was used.

\section{CLASSIFICATION OF PANCREATIC LESIONS}

To clarify presentation of the results, three subsets of pancreatic neoplasms are distinguished: 1 , adenocarcinoma; 2 , neuroendocrine tumour; 3, various premalignant neoplasms. Premalignant exocrine neoplasms - for example, intraductal papillary mucinous tumours and solid pseudo-papillary tumourwere classified in group 3. In some cases, the diagnosis was only considered to be "compatible with" a malignant tumour by the pathologist when definitive criteria were lacking.

STATISTICAL ANALYSIS

The $\chi^{2}$ and Fisher exact tests were used for comparisons. When diagnostic accuracy was considered, patients in whom EUS-FNAB was impossible because of technical problems were excluded. Diagnostic accuracy was defined as the ratio between the sum of true positive and true negative values divided by the number of lesions. Positive and negative predictive values were calculated for distinction between pancreatic neoplasms and chronic pancreatitis, as only the former theoretically require resection.

\section{Results}

EUS-FNAB was successful in 90 patients (91\%). Uncomplicated procedures were performed in six patients with portal hypertension and in seven with pancreatic calcifications. Technical failure occurred in nine patients. In four patients, proximity of vascular structures located in the gastrointestinal wall prevented the passage of the biopsy needle. In two patients, the tumour was too hard for needle penetration. These tumours were located in the uncinate process with the endoscope in the second part of the duodenum. This "long position" prevented adequate force from being applied for tissue penetration. In one patient, duodenal stenosis prevented passage of the instrument, and in another, the duodenum was excessively mobile which prevented correct positioning. In a further patient, biopsy of the mass proved impossible as the needle failed to advance into the target tissue. This tumour was located in the isthmus, with the endoscope positioned in the gastric antrum, again in the long position preventing sufficient force for needle penetration of the tumour.

Complete records were available for all 90 patients having successful EUS-FNAB. There were $52(58 \%)$ men and $38(42 \%)$ women. Mean age was 59.6 years. The site of the lesion was as follows: pancreatic head, $\mathrm{n}=52(58 \%)$; isthmus, $\mathrm{n}=9(10 \%)$; body, $\mathrm{n}=11(13 \%)$; tail, $\mathrm{n}=3(3 \%)$; extending to more than one site, $\mathrm{n}$ $=12(13 \%) ;$ multifocal, $\mathrm{n}=3(3 \%)$. The mean size of the lesions was 34 (range 6-100) $\mathrm{mm}$ and the mean number of needle passes was 2.7 (range one to six).

Confirmation of the EUS-FNAB histological diagnosis was available in 31 patients: 25 after surgical resection; two from intraoperative biopsy; two from biopsy of another tumour site; two from autopsy. The final diagnosis was assessed using a combination of clinical, biological, and imaging criteria as stated in the Methods section in the 59 remaining patients.

\section{COMPLICATIONS}

Complications occurred in five patients (5\%). In four patients, bleeding was noted during the procedure. In three of them, this consisted of a small rim of fluid and, in one, a $3 \mathrm{~cm}$ stable duodenal haematoma. Bleeding was not apparent clinically and no patient required blood transfusion. One patient was readmitted the day after the procedure with abdominal pain and pyrexia, but was found to have normal serum amylase level and no collection on CT scan; symptoms resolved spontaneously. Mild abdominal discomfort occurred occasionally after the procedure and responded to simple analgesics. There was no case of acute pancreatitis.

\section{HISTOLOGICAL RESULTS}

Table 1 shows a comparison of the final diagnosis with the histological diagnosis obtained by EUS-FNAB. In five patients, adenocarcinoma had developed from an intraductal papillary mucinous tumour. In two others, pancreatic tumours were metastases from a renal clear cell cancer $(n=1)$ or a breast cancer $(n=1)$. One patient had an osteoclast-like adenocarcinoma.

In 17 cases $(19 \%)$, the specimens obtained were purely haemorrhagic and could not be used for analysis as no pancreatic tissue was obtained. These results were found in $4 / 21$ (19\%) tumours less than $25 \mathrm{~mm}$ in diameter and in 13/69 (19\%) tumours larger than 25 $\mathrm{mm}$. Seven of the 15 neuroendocrine tumours $(47 \%)$ were non-informative, but this figure was only $8 / 59(14 \%)$ in adenocarcinomas $(p<0.01)$. Figures $3-6$ show examples of histological results.

Overall diagnostic accuracy in analysable cases was $74 \%$ (table 1). It was more accurate for adenocarcinomas than for neuroendocrine tumours $(81 \% v 47 \%, \mathrm{p}<0.02)$, and $75 \%$ for the remaining lesions. The sensitivity, specificity, and positive and negative predictive values in diagnosing a pancreatic neoplasm (exocrine or endocrine) were $75 \%, 87.5 \%, 98.4 \%$, and $25.6 \%$ respectively. When neuroendocrine 


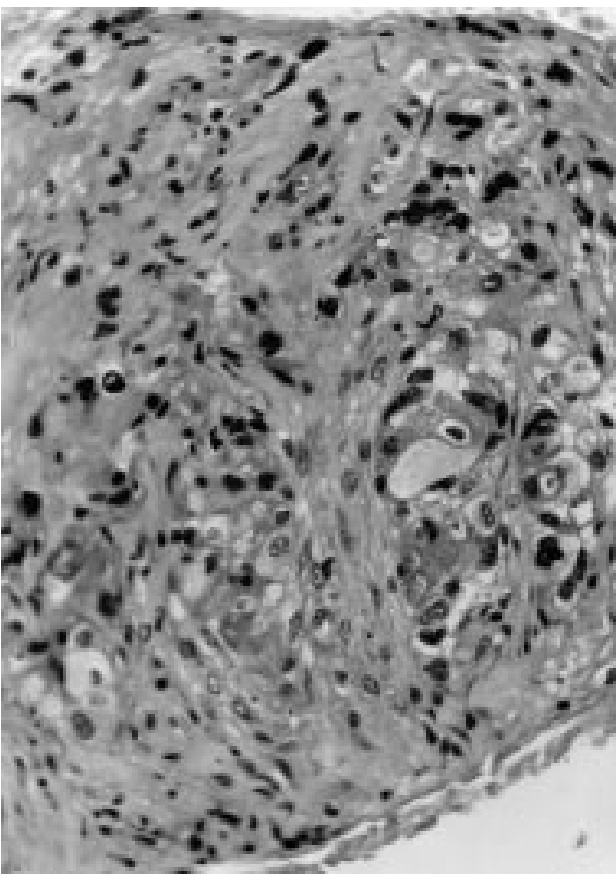

Figure 3 Large fragment of poorly differentiated ductal adenocarcinoma obtained using endoscopic ultrasound guided fine needle aspiration biopsy showing sheets of tumour cells with severe cytological atypia. Note the abundant fibrous stroma. Haematoxylin-eosin stain, original magnification $\times 360$.

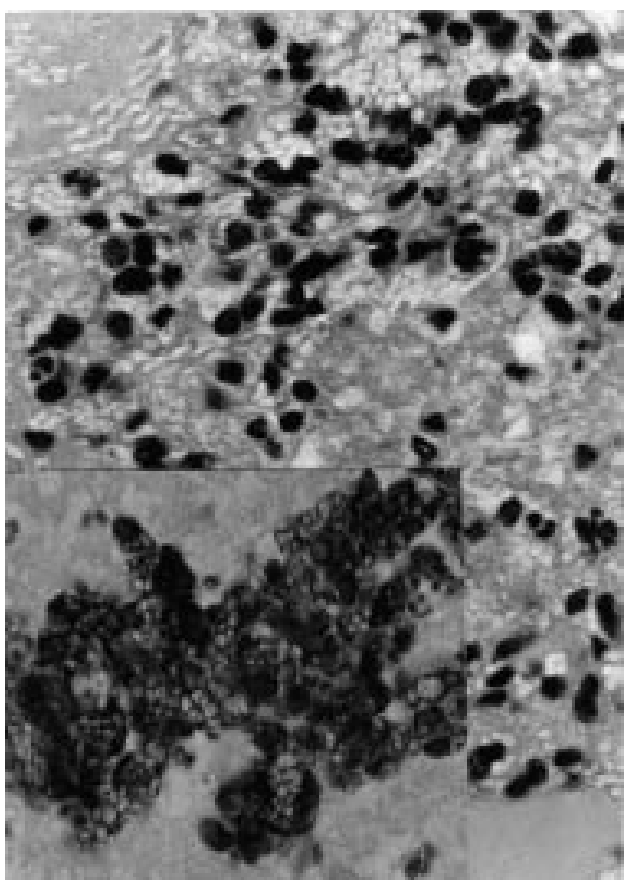

Figure 4 Cluster of monomorphous and regular small cells, without glandular or neuroendocrine structures, stained by the immunoperoxidase technique for $K L 1$ (inset). The diagnosis of solid pseudopapillary and cystic tumour was proposed in view of the clear aspect of the cytoplasm.

tumours were excluded from the analysis, the sensitivity, specificity, and positive and negative predictive values in the differentiation between all exocrine neoplasms and chronic pancreatitis were $81.6 \%, 87.5 \%, 98.1 \%$, and $36.8 \%$ respectively. Finally, the sensitivity, specificity, and positive and negative predictive values in

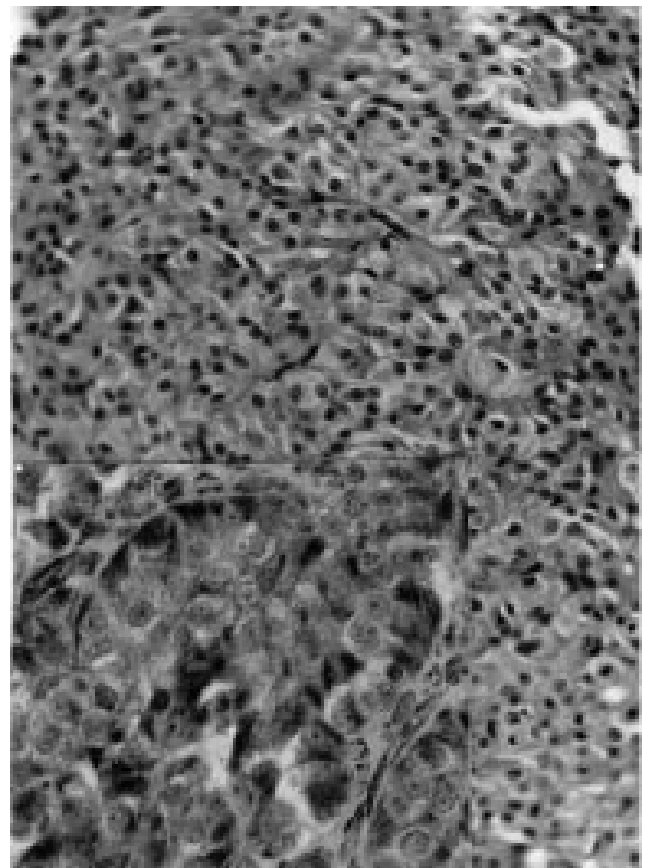

Figure 5 Large core biopsy illustrating a neuroendocrine tumour composed of ribbons of uniform small cells with

fibrovascular stroma and staining positive for chromogranin A (inset). Haematoxylin-eosin stain; original magnification $\times 360$; original magnification of the inset $\times 720$.

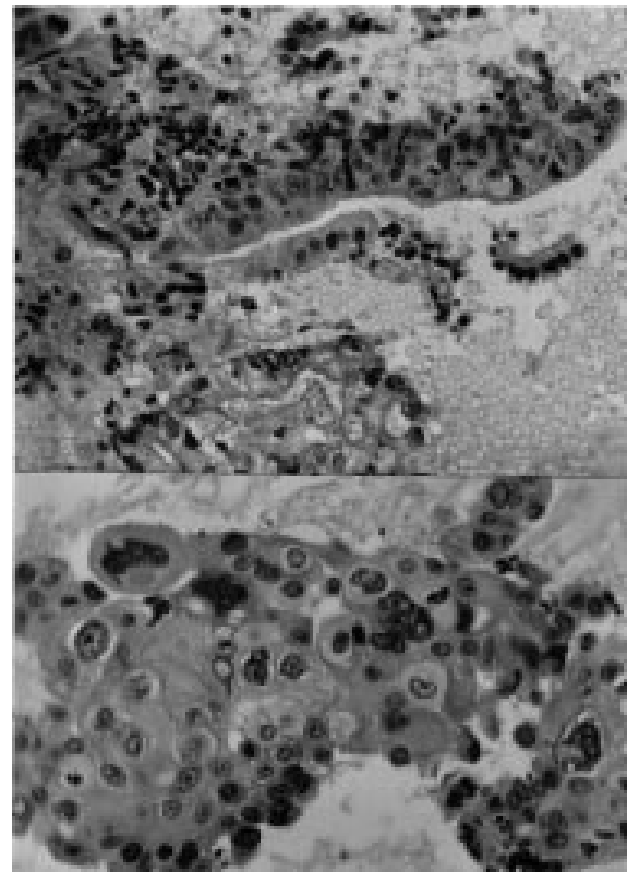

Figure 6 Two cases of endoscopic ultrasound guided fine needle aspiration biopsy showing small tumour fragment samples. Top, shreds of epithelial cells with moderate cytological atypia compatible with intraductal papillary mucinous tumour of the pancreas; bottom, poorly

differentiated adenocarcinoma. Haematoxylin-eosin stain; original magnification $\times 360$.

the differentiation between adenocarcinoma and chronic pancreatitis were $81.4 \%, 87.5 \%$, $98 \%$, and $38.9 \%$ respectively.

EUS-FNAB proved to be falsely positive for carcinoma in one patient whose biopsy showed both acinar involution with atypical cells and trabecular patterns. However, histological 
analysis of the resected specimen after pancreaticoduodenectomy showed histological changes consistent with chronic pancreatitis with no evidence of malignancy. Interestingly, this patient was also adjudged to have a malignant tumour on CT scan, EUS, and endoscopic retrograde cholangiopancreatography examinations with an irregular cephalic stenosis of the main pancreatic duct and a well defined $3 \mathrm{~cm}$ mass. When technical failure at biopsy, non-informative samples, and failure at histology were pooled, the global accuracy rate for all 99 patients was $68 \%$.

\section{Discussion}

In this study, EUS-FNAB was successful in all patients but nine. Specimens obtained were analysable in $81 \%$ of cases. This result is slightly lower than the 88 to $90 \%$ previously reported $^{11216-19}$ These studies included lymph nodes, which are known to yield higher success rates. Two recent reports on EUS-FNAB in pancreatic tumours reported similar results to ours. $^{2021}$ Pancreatic tumours located in the isthmus and uncinate process prove more difficult to biopsy at EUS than in other locations, as the scope is often positioned in the long position which decreases the necessary force and axis of tissue penetration. The presence of prominent stromal fibrosis in such tumours may also interfere with success at needle puncture. Possible technical solutions to increase success at biopsy include: the use of a larger diameter needle as defined by Binmoeller and colleagues $^{20}$; the use of an instrument that allows penetration of the biopsy needle (22 gauge) at a more open angle (guided with an elevator) almost perpendicular to the tumour (Palazzo et al, unpublished data), which appears promising for tumours located in the head and uncinate process; the use of an automated biopsy device to increase the biopsy success rate in the case of indurated lesions. ${ }^{22}$

In contrast with other reports, ${ }^{72} 1621$ in this study a cytopathologist was not present during the procedure to ensure adequate cellularity of the specimens. Using this procedure, we obtained an adequate tissue sample for histological analysis, therefore obviating the need for on site presence of a pathologist. Tumour changes such as necrosis, desmoplastic reaction, and heterogeneity may influence the histological yield. The needle used for EUSFNAB is of a smaller calibre (22 gauge) than that used for radiologically guided biopsies (18 or 19 gauge); this may result in a smaller proportion of diagnostic core needle biopsies. In contrast with a previous study showing that histology was less informative for solid tumours larger than $4 \mathrm{~cm}$ as the result of necrosis, ${ }^{17}$ we found that EUS-FNAB accuracy was comparable in small and large tumours. On the other hand, the high resolution of EUS allowed localisation and aspiration of small lesions (as small as $6 \mathrm{~mm}$ in this study), and the rate of contributive samples obtained was similar whether the lesion size was smaller or larger than $25 \mathrm{~mm}$. As EUS is also accurate in detecting enlarged venous collaterals in portal hypertension, ${ }^{23}$ EUS guidance of the needle to areas free of varices should decrease the risk of bleeding after biopsy. In our study, six patients with portal hypertension had uncomplicated procedures. None of the patients in our study developed severe complications of FNAB which include acute pancreatitis, significant bleeding, and perforation.

The positive predictive value of EUS-FNAB in differentiating between pancreatic neoplasm and pancreatitis was high in this series (98\%). The single false positive case of pancreatic adenocarcinoma in a patient with chronic pancreatitis is a cause for concern but is not unique to this procedure. This is likely to be a function of the nature of cellular changes occurring in patients with chronic pancreatitis. It would appear that the diagnostic ability of EUSFNAB depends on the histological nature of the tumour. Less diagnostic accuracy was obtained for neuroendocrine tumours than for adenocarcinomas. Specimens obtained from the former were often haemorrhagic possibly because of their hypervascularisation, even if no bleeding was seen after the procedure. Perhaps, new cytological methods including the ThinPrep procedure may enhance results obtained using cytology in such lesions. ${ }^{24}$ Endoscopic retrograde cholangiopancreatography with cytology of pancreatic juice has been reported to give a diagnostic accuracy of up to $56 \%$ in pancreatic carcinoma, ${ }^{25}{ }^{26}$ but, unlike EUS, this method does not define the morphology of the lesion and the tumour staging. Moreover, it is accompanied by a 3\% incidence of acute pancreatitis. ${ }^{27}$ A recently reported new procedure, pancreatoscopy with biopsy, ${ }^{28}$ shows promise in the tissue diagnosis of ductal lesions, but again does not contribute to the tumour staging. The efficacy of radiologically guided transperitoneal FNAB of the pancreas is well established. ${ }^{29-31}$ The risks of transperitoneal tumour seeding are ill defined. ${ }^{32-39}$ Lesions in the pancreatic head may be biopsied using EUS-FNAB through the duodenum, theoretically without any risk of transperitoneal seeding, the needle tract being removed with the surgical specimen. Lesions in the body and tail require needle passage through the lesser sac with a theoretical risk of tumour seeding. Therefore in patients with potentially resectable lesions of the pancreatic body or tail, the risk/benefit ratio of EUSFNAB should be carefully considered.

In conclusion, EUS-FNAB is shown to be an effective procedure in establishing a tissue diagnosis in selected patients with pancreatic lesions, some of whom do not require surgical management. We show that EUS-FNAB is feasible and accurate in the diagnosis of pancreatic masses even when tumour size is small. The complication rate of this technique was negligible even in patients with portal hypertension. In the near future, technical advances in echoendoscopes and improved histological and cytological methods will increase further the success of EUS-FNAB and decrease the number of non-informative specimen samples. 
We thank Drs J Belghiti, O Farges, and A Sauvanet, who performed the pancreatic resections, and Dr F Pessione for her performed the pancreatic resectio
help in the statistical analysis.

1 Wanebo HJ, Vezeridis MP. Pancreatic carcinoma in perspective. A continuing challenge. Cancer perspective. A co $1996 ; 78$ (suppl): $580-90$.

2 Raymond E, de Gramont A, Louvet C, et al. Clinical benefit with cisplatin, hydroxyurea and 5 fluorouracil/leucovorin in with cisplatin, hydroxyurea and 5 fluorouracil/leucovorin in advanced pan

3 André T, Lotz JP, Bouleuc C, et al. Phase II trial of 5-fluorouracil, leucovorin and cisplatin for treatment of advanced pancreatic adenocarcinoma. Ann Oncol 1996;7: $173-8$

4 Lee YT. Tissue diagnosis for carcinoma of the pancreas and periampullary structures. Cancer 1982;49:1035-9.

5 Lux G, Heyder N, Lutz H, et al. Endoscopic ultrasonography: technique, orientation and diagnostic possibilities. Endoscopy 1982;14:220-5.

6 Tio TL, Tytgat GN. Endoscopic ultrasonography in the assessment of intra and transmural infiltration of tumours in the oesophagus, stomach and papilla of Vater and in the in the oesophagus, stomach and papilla of Vater and in the detection

7 Chang KJ. Endoscopic ultrasound: moving towards permanence. Gastrointest Endosc 1996;44:502-4.

8 Tytgat GNJ, Fockens P. Exploring the role of endosonography. Scand F Gastroenterol 1996;31 (suppl 20):71-4.

9 Palazzo L. Staging of pancreatic carcinoma by endoscopic ultrasonography. Endoscopy 1998;30(suppl 1):103-7.

10 Vilmann P, Hancke S, Fenriksen FW, et al. Endosonographically guided fine needle aspiration biopsy of malignan lesions in the upper gastrointestinal tract. Endoscopy 1993; 25:523-7.

11 Giovannini M, Seitz JF, Monges G, et al. Fine needle aspiration cytology guided by endoscopic ultrasonography: results in 141 patients. Endoscopy 1995;27:171-7.

12 Wiersema MJ, Vilmann P, Giovannini M, et al. Endoscopicguided fine-needle aspiration biopsy: diagnostic accuracy and complication assessment. Gastroenterology 1997;112: and comp 1085 .

13 MacCarthy R L. Cyst fluid analysis and imaging of pancreatic cystic lesions. AfR Am 7 Roentgenol 1995;164:820-1.

14 Lewandrowski KB, Southern JF, Pins MR, et al. Cyst fluid Lewandrowski KB, Southern JF, Pins MR, et al. Cyst fluid
analysis in the differential diagnosis of pancreatic cysts. analysis in the differential
Ann Surg 1993;217:41-7.

15 Hammel P, Lévy P, Voitot H, et al. Preoperative cyst fluid analysis is useful for the differential diagnosis of cystic lesions of the pancreas. Gastroenterology 1995;108: 1230-5.

16 Gress FG, Hawes RH, Savides TJ, et al. Endoscopic ultrasound-guided fine needle aspiration biopsy using linear array and radial scanning endosonography. Gastrointest Endosc 1997;45:243-50.

17 Giovannini M. Ponction biopsie guidée sous échoendoscopie. Gastroenterol Clin Biol 1997;21:355-7.

18 Chang KJ, Katz KD, Durbin TE, et al. Endoscopic ultrasound-guided fine needle aspiration. Gastrointest Endosc 1994;40:694-9.

19 Cahn M, Chang K, Nguyen P, et al. Impact of endoscopic ultrasound with fine-needle aspiration of the surgical
management of pancreatic cancer. Am f Surg 1996;172: man-2.
20 Binmoeller KF, Thul R, Rathod V, et al. Endoscopic ultrasound-guided, 18-gauge, fine needle aspiration biopsy
of the pancreas using a $2.8 \mathrm{~mm}$ channel convex array echof the pancreas using a $2.8 \mathrm{~mm}$ channel convex

21 Bhutani MS, Hawes RH, Baron PL, et al. Endoscopic ultraound guided fine needle aspiration of malignant pancreatic lesions. Endoscopy 1997;29:854-8.

22 Binmoeller KF, Jabusch HC, Seifert $\mathrm{H}$, et al. Endosonography-guided fine-needle biopsy of indurated pancreatic lesions using an automated biopsy device. Endoscopy 1997;29:384-8

23 Boustière C, Dumas O, Joufre C, et al. Endoscopic ultrasonography classification of gastric varices in patients with cirrhosis. Comparison with endoscopic findings. $\mathcal{F}$ Hepatol 1993;19:268-72.

24 Fabre M, Monges G, Ben Lagha N, et al. Paired comparison of conventional and Thinprep fine needle aspiration (FNA) biopsy. A prospective series of 42 deep masses [abstract]. Arch Anat Cytol Pathol 1998;46:A238.

25 Kasugai T, Kobayashi S, Kuno N. Endoscopic cytology of the oesophagus, stomach and pancreas. Acta Cytologica 1978:22;327-30.

26 Shoefl R, Haefner M, Wrba F, et al. Forceps biopsy and brush cytology during endoscopic retrograde cholangiopancreatography for the diagnosis of biliary stenoses. Scand 7 Gastroenterol 1997;32:363-8.

27 Cotton P, Williams C. Practical gastrointestinal endoscopy. Oxford: Blackwell, 1996:169.

28 Uehara H, Nakaizumi A, Iishi $\mathrm{H}$, et al. Diagnosis of carcinoma in situ of the pancreas by per oral pancreatoscopy carcinoma in situ of the pancreas by per oral pancreatosco

29 Waldner B, Dorval ED, Anthonioz P, et al. Cytoponction pancréatique à l'aiguille fine. A propos de 45 observations. Gastroenterol Clin Biol 1986;10:545-8.

30 Celle G, Savarino V Biggi E, et al. Fine-needle aspiration cytodiagnosis: a simple and safe procedure for cancer of the pancreas. Gastroenterol Clin Biol 1986;10:545-8.

31 Greenberg ML, Pancreas. In: Grey W, ed. Diagnostic cytopathology. Edinburgh: Churchill Livingstone, 1995: 415-33.

32 Ferrucci JT, Wittenberg J, Margolies $\mathrm{MN}$, et al. Malignant seeding of the needle tract after fine needle aspiration biopsy. Radiology 1979;130:345-6.

33 Smith FP, MacDonald JS, Schein PS, et al. Cutaneous seeding of pancreatic cancer by skinny needle aspiration biopsy. Arch Intern Med 1980;140:855.

34 Rashleigh-Belcher HJ, Russel RC, Lees WR. Cutaneous implantation metastases after fine needle puncture of a pancreatic cancer. Gastrointestinal Radiology 1986;11:81-4.

35 Frölich E, Frühmorgen P, Seelingher H. Cutaneous implantation metastases after fine needle puncture of a pancreatic cancer. Ultraschall Med 1986;7:141-4.

36 Hall-Craggs MA, Lees WF. Fine needle aspiration biopsy: pancreatic and biliary tumours. AfR Am f Roentgenol 1986; 14:399-403.

37 Morgenfeldt M, Genell S, Lindholm K, et al. Needle tract seeding after percutaneous fine needle biopsy of pancreatic carcinoma. Case report. Scand 7 Gastroenterol 1989;24: 949-55

38 Warshaw AL. Implications of peritoneal cytology for staging of early pancreatic cancer. Am $\mathcal{F}$ Surg 1991;161:26-30.

39 Leach SD, Rose JA, Lowy AM, et al. Significance of peritoneal cytology in patients with potentially resectable adenocarcinoma of the pancreatic head. Surgery 1995;118:472-8. 